\title{
Consenso Chileno 2005: Manejo de la neumonía del adulto adquirida en la comunidad Introducción
}

FERNANDO SALDÍAS P. y CARLOS PÉREZ C.

\author{
National consensus for management of community acquired \\ pneumonia in adults \\ Introduction
}

Community acquired pneumonia (CAP) is an acute respiratory infection that affects pulmonary parenchyma, and is caused by community acquired microorganisms. In Chile, pneumonia represents the main cause of death due to infectious diseases and is the third specific cause of mortality in adults. In 1999, an experts committee in representation of "Sociedad Chilena de Enfermedades Respiratorias", presented the first National Guidelines for the Treatment of Adult Community Acquired Pneumonia, mainly based in foreign experience and documents, and adapted it to our National Health System Organization. During the last decade, impressive epidemiological and technological changes have occurred, making the update of guidelines for treatment of NAC by several international scientific societies, necessary. These changes include: new respiratory pathogens that are being identified in CAP and affect adult patients (Mycoplasma pneumoniae, Chlamydia pneumoniae, Legionella pneumophila); the increasing senescent adult population that carries multiple co-morbidities; the emergence of antimicrobial resistance among respiratory pathogens associated to massive antibiotic prescription; the development by the pharmaceutical industry of new drugs that are effective for pneumonia treatment (macrolides, ketolides and respiratory fluorquinolones); and the development of new diagnostic techniques for detection of antigens, antibodies, and bacterial DNA by molecular biology, useful in respiratory infections. Based on these antecedents, an Advisory Committee of "Sociedad Chilena de Enfermedades Respiratorias" and "Sociedad Chilena de Infectología" has reviewed the national and international evidence about CAP management in adults in order to update clinical recommendations for our country.

Key words: community-acquired infections, pneumonia, guidelines.

Palabras clave: Infecciones adquiridas en la comunidad, neumonía, guías clínicas.

La neumonía adquirida en la comunidad (NAC) es la infección que compromete el parénquima pulmonar producida por la invasión de microorganismos de adquisición extrahospitalaria. La gravedad de la infección respiratoria varía desde cuadros leves en personas sanas, que pueden confundirse con resfríos, bronquitis o infecciones de la vía aérea superior, hasta cuadros graves que requieren admisión a unidades especializadas de cuidado intensivo y ponen en peligro la vida de los pacientes.
La NAC del adulto es una patología infecciosa prevalente, se estima que representa el 3 a $5 \%$ de las consultas por enfermedades respiratorias en los servicios de atención primaria (consultorios y servicios de urgencia), y su incidencia va en aumento en nuestro país asociado al envejecimiento de la población y al aumento de las enfermedades crónicas (cardiopatía coronaria, insuficiencia cardíaca, EPOC, enfermedad cerebrovascular, insuficiencia renal crónica, diabetes mellitus, neoplasias) ${ }^{1-3}$. En Chile, la neumo-

Coordinador de la Comisión de Consenso de Neumonía del Adulto Adquirida en la Comunidad. Sociedad Chilena de Enfermedades Respiratorias (FSP).

Presidente de la Sociedad Chilena de Infectología (CPC). 
nía es la principal causa de muerte por enfermedades infecciosas y la tercera causa específica de muerte en la población adulta ${ }^{3}$. El aumento de la demanda de recursos de salud, pérdida de productividad de la población adulta y letalidad asociados a esta patología, han determinado que sea considerada un grave problema de salud pública en el ámbito mundial.

Reconociendo la importancia de la NAC en la población adulta, numerosos países han elaborado guías clínicas con el propósito de racionalizar el manejo de los enfermos, siendo más conocidas en el medio nacional las guías clínicas de la Sociedad Americana de Tórax (American Thoracic Society - ATS) ${ }^{4}$, la Sociedad Británica de Tórax (British Thoracic SocietyBTS $)^{5}$ y la Sociedad Americana de Enfermedades Infecciosas (Infectious Diseases Society of America-IDSA) ${ }^{6}$. En 1999, un comité de expertos de la Sociedad Chilena de Enfermedades Respiratorias elaboró la primera guía nacional de manejo de la NAC del adulto, basado fundamentalmente en la experiencia extranjera adaptada a la organización del sistema de salud de nuestro país ${ }^{7}$. En la última década, han ocurrido cambios epidemiológicos y tecnológicos significativos que han obligado a las sociedades científicas internacionales a actualizar las guías clínicas para el manejo de la neumonía.

Los principales cambios epidemiológicos acontecidos en la última década que justifican la actualización de las guías clínicas nacionales son:

- El reconocimiento de nuevos patógenos respiratorios involucrados en la NAC del adulto, tales como Mycoplasma pneumoniae, Chlamydia pneumoniae, Legionella pneumophila, y los virus respiratorios.

- El aumento progresivo de la población senescente con comorbilidad múltiple, lo que favorece la infección por microorganismos resistentes a los antimicrobianos, especialmente los bacilos gramnegativos.

- La emergencia de patógenos respiratorios resistentes a los antimicrobianos asociado al uso indiscriminado de antibióticos en infecciones respiratorias virales autolimitadas tales como resfríos, faringitis, laringitis, bronquitis aguda e influenza. En Chile, las tasas de resistencia de Streptococcus pneumoniae a penicilina fluctúan entre 20 y $30 \%$ y a macrólidos entre 10 y $15 \% \%^{8,9}$.

- El desarrollo de nuevos antimicrobianos por la industria farmacéutica efectivos para el tratamiento de la NAC, tales como los nuevos macrólidos, cetólidos y fluoroquinolonas. En esta guía se examinará sólo la evidencia rela- cionada con los antimicrobianos disponibles en el medio nacional.

- El desarrollo de nuevas técnicas de detección de antígenos, serología y biología molecular disponibles en el medio nacional para el diagnóstico de las infecciones respiratorias por patógenos bacterianos, virales y microorganismos atípicos. A modo de ejemplo, la detección del antígeno urinario ha facilitado el diagnóstico de la infección por L. pneumophila ${ }^{10}$ y $S$. pneumoniae ${ }^{11}$.

- En la última década, se han publicado varios estudios clínicos en el medio nacional que han examinado y actualizado la epidemiología, etiología, cuadro clínico, factores pronósticos y tratamiento de la NAC del adulto.

El comité de expertos de la Sociedad Chilena de Enfermedades Respiratorias en conjunto con miembros de la Sociedad Chilena de Infectología y Sociedad Chilena de Medicina Intensiva han revisado minuciosamente la evidencia nacional y extranjera concerniente con el manejo de la NAC del adulto previo a elaborar las actuales recomendaciones clínicas que tienen por objetivo ser aplicadas en el medio nacional.

\section{Objetivos}

- Las Guías Clínicas de la Sociedad Chilena de Enfermedades Respiratorias y la Sociedad Chilena de Infectología tienen por objeto revisar el manejo ambulatorio y hospitalizado de los adultos inmunocompetentes que consultan por NAC.

- Esta Guía Clínica no examina el manejo de los enfermos con deterioro significativo de su sistema inmune, excluyendo a los pacientes portadores de una neoplasia activa, inmunodeficiencia primaria o secundaria, quimioterapia, etc, considerando que el manejo de los enfermos inmunocomprometidos difiere significativamente en complejidad y tiene características clínicas particulares.

- Las Guías Clínicas no consideran el manejo de la neumonía adquirida en el hospital y la neumonía comunitaria en la población pediátrica.

- La metodología, nivel de evidencia y graduación de las recomendaciones clínicas se ha realizado siguiendo una pauta preestablecida definida por el comité de expertos.

- Las Guías Clínicas de Manejo de la Neumonía Comunitaria del Adulto Inmunocompetente están destinadas a ser aplicadas en el Sistema Público y Privado de nuestro país. 
En el desarrollo de estas normativas se ha utilizado un enfoque basado en la evidencia tras el análisis de los estudios relevantes publicados hasta la fecha de redacción del documento, con énfasis en el análisis de las publicaciones sobre el tema aparecidas en el medio nacional. Para clasificar la evidencia que sostiene las recomendaciones se han utilizado cuatro niveles ${ }^{5}$ : Evidencia de nivel I, a partir de estudios clínicos prospectivos controlados y aleatorios que responden la pregunta de interés; evidencia de nivel II, a partir de estudios prospectivos bien diseñados y controlados pero no aleatorios (incluyen estudios de cohortes, series de pacientes y estudios de caso y control); evidencia de nivel III, a partir de estudios clínicos retrospectivos que ilustran sobre el problema pero no responden rigurosamente la pregunta clínica planteada; y evidencia de nivel IV, a partir de series de casos y opinión de expertos. Cuando las recomendaciones de tratamiento procedan de datos microbiológicos sobre susceptibilidad antimicrobiana, sin observaciones clínicas, se catalogarán como evidencia de nivel IV (Tabla 1).

\section{Resumen}

La neumonía adquirida en la comunidad (NAC) es la infección respiratoria aguda que compromete el parénquima pulmonar, ocasionada por micro-organismos de adquisición extrahospitalaria. En Chile, la neumonía es la principal causa de muerte por enfermedades infecciosas y la tercera causa específica de muerte en la población adulta. En 1999, un comité de expertos de la Sociedad Chilena de Enfermedades Respiratorias elaboró la primera guía nacional de manejo de la neumonía del adulto adquirida en la comunidad, basada fundamentalmente en la experiencia extranjera y adaptada al sistema de salud de nuestro país. En la última década, cambios epidemiológicos y tecnológicos significativos han impulsado a las sociedades científicas internacionales a actualizar las guías clínicas de neumonía, tales como la identificación de nuevos patógenos respiratorios involucrados en la neumonía del adulto (Mycoplasma pneumoniae, Chlamydia pneumoniae, Legionella pneumophila); el aumento de la población senescente con co-morbilidad múltiple; la emergencia de patógenos respiratorios resistentes a los antimicrobianos asociada al uso indiscriminado de antibióticos; el desarrollo de nuevos antimicrobianos por la industria farmacéutica efectivos para el tratamiento de la neumonía (macrólidos, cetólidos y fluoroquinolonas); y el desarrollo de nuevas técnicas de detección de antígenos, serología y biología molecular para el diagnóstico de las infecciones respiratorias. Basados en estos antecedentes, un comité de expertos de la Sociedad Chilena de Enfermedades Respiratorias y de la Sociedad Chilena de Infectología ha revisado la evidencia nacional y extranjera concerniente al manejo de la neumonía del adulto adquirida en la comunidad, previo a elaborar las actuales recomendaciones clínicas que tienen por objetivo ser aplicadas en el medio nacional.

\section{Bibliografía}

1.- Metlay J P, Stafford R S, Singer D E. National trends in the management of acute cough by primary care physicians. J Gen Intern Med 1997; 12 (Suppl): 77.

2.- Diehr P, Wood R W, Bushyhead J, Krueger L, Wolcott $\mathrm{B}$, Tompkins $\mathrm{R} \mathrm{K}$. Prediction of pneumonia in outpatients with acute cough- a statistical approach. J Chron Dis 1984; 37: 215-25.

3.- Misterio de salud de Chile. Departamento de estadísticas e información de salud (http://deis. minsal.cl/ index.asp).

4.- Niederman M S, Mandell L A, Anzueto A, Bass J B, Broughton W A, Campbell G D, et al. Guidelines for the management of adults with community-acquired pneumonia. Diagnosis, assessment of severity, antimicrobial therapy, and prevention. Am J Respir Crit Care Med 2001; 163: 1730-54.

5.- British Thoracic Society Standards of Care Committee. British Thoracic Society guidelines for the management of community-acquired pneumonia in adults. Thorax 2001; 56 (Suppl IV): 1-64.

6.- Mandell L A, Bartlett J G, Dowell S F, File T M Jr, Musher D M, Whitney C. Update of practice guidelines for the management of community-acquired pneumonia in immunocompetent adults. Clin Infect Dis 2003; 37: 1405-33.

7.- Sociedad Chilena de Enfermedades Respiratorias. Consenso Nacional en Neumonías Adquiridas en la Comunidad en Adultos y Niños. Rev Chil Enf Respir 1999; 15: 67-136.

8.- Castanheira M, Gales A C, Mendes R E, Jones R N, Sader H S. Antimicrobial susceptibility of Streptococcus pneumoniae in Latin America: results from five years of the SENTRY Antimicrobial Surveillance Program. Clin Microbiol Infect 2004; 10: 645-51.

9.- Saldías F, Flores L J, Torres C, García P, Díaz A. Susceptibilidad a antimicrobianos de Streptococcus pneumoniae en población infantil y adulta de Santiago. Periodo 1997-2003. Rev Méd Chile 2005; 133: 42-9.

10.- Cabello H, Cortés C, Ruíz M, Jover E, Rivera F, Segovia E, et al. Neumonía adquirida en la comunidad. Comunicación de 8 casos de neumonía grave por Legionella pneumophila serogrupo 1 en Chile. Rev Méd Chile 2002; 130: 309-13.

11.- Ishida T, Hashimoto T, Arita M, Tojo Y, Tachibana H, Jinnai M. A 3-year prospective study of a urinary antigen-detection test for Streptococcus pneumoniae in community-acquired pneumonia: utility and clinical impact on the reported etiology. J Infect Chemother 2004; 10: 359-63. 
Tabla 1. Definición del nivel de evidencia y recomendación empleados en el Consenso Nacional del Adulto adquirida en la comunidad

\begin{tabular}{|c|c|c|}
\hline Nivel de evidencia & Definición & Recomendación \\
\hline Ia & $\begin{array}{l}\text { Revisión sistemática de estudios diseñados para } \\
\text { responder la pregunta de interés }\end{array}$ & A+ \\
\hline $\mathrm{Ib}$ & $\begin{array}{l}\text { Uno o más estudios clínicos prospectivos } \\
\text { controlados y aleatorios que responden la } \\
\text { pregunta de interés }\end{array}$ & A- \\
\hline II & $\begin{array}{l}\text { Uno o más estudios clínicos prospectivos bien } \\
\text { diseñados y controlados pero no aleatorios, que } \\
\text { ilustran pero no responden rigurosamente la } \\
\text { pregunta de interés }\end{array}$ & B \\
\hline III & $\begin{array}{l}\text { Uno o más estudios clínicos retrospectivos que } \\
\text { ilustran pero no responden rigurosamente la } \\
\text { pregunta clínica de interés }\end{array}$ & $\mathrm{C}$ \\
\hline IV & $\begin{array}{l}\text { Opinión de expertos, series de casos u otra fuente } \\
\text { de información }\end{array}$ & $\mathrm{D}$ \\
\hline
\end{tabular}

Correspondencia a:

Fernando Saldías P.

fsaldias@med.puc.cl

Carlos Pérez C.

cperez@med.puc.cl 